\title{
Re: Outcomes of Microdissection Testicular Sperm Extraction in Men with Nonobstructive Azoospermia Due to Maturation Arrest
}

\author{
Aaron M. Bernie1, Kalee Shah1, Joshua A. Halpern1, Jason Scovell2, Ranjith Ramasamy2, \\ Brian Robinson3, Peter Schlegel4 \\ 1 Weill Cornell Medical College, Department of Urology, New York, USA \\ 2 Baylor College of Medicine, Texas, USA \\ 3 Weill Cornell Medical College, Department of Pathology\&tLaboratory Medicine, New York, USA \\ 4 Weill Cornell Medical College, Department of Urology, New York, USA
}

Fertil Steril 2015;104:569:573. e1. doi: 10.1016/j.fertnstert.2015.05.037. Epub 2015 Jun 18.

\section{EDITORIAL COMMENT}

Sperm extraction from testicular tissue in men with nonobstructive azoospermia (NOA) due to maturation arrest (MA) is one of the most challenging procedures. Particularly in these patients, determination of potential sperm recovery before the microdissection testicular sperm extraction (micro TESE) can be complex. In this article, the authors tried to answer the question whether any clinical data can predict sperm recovery in special subgroup of men with NOA. They retrospectively analyzed the records of 211 patients with MA who underwent micro TESE. All over sperm retrieval rate was 52\%, however, in patients with late MA pattern and higher FSH levels, significantly higher sperm recovery rate were observed compared to those with early MA and close to normal FSH levels. In addition, they found that a history of cryptorchidism and focal MA rather than diffuse MA was positively associated with sperm recovery. This study showed that the chance of sperm recovery is the worst if an azoospermic man is with normal testis volume, normal FSH levels and diffuse MA pathology.

Emre Bakırcıoğlu, MD

\section{Re: Long-term Exposure to Testosterone Therapy and the Risk of High Grade Prostate Cancer}

\author{
Baillargeon Jacques1, Kuo Yong-Fang1, Fang Xiao2, Shahinian Vahakn B3 \\ 1 University of Texas Medical Branch, Sealy Center on Aging, Department of Internal Medicine, Texas, USA \\ 2University of Texas Medical Branch, Department of Preventive Medicine and Community Health, Texas, USA \\ 3University of Michigan, Department of Internal Medicine, Michigan, USA
}

J Urol 2015. pii: S0022-5347(15)04148-8. doi: 10.1016/j.juro.2015.05.099. [Epub ahead of print]

\section{EDITORIAL COMMENT}

Late, -onset hypogonadism in men has been increasingly diagnosed and exogenous testosterone treatment has been used over a decade. Despite this rapid growth, there have been no large, long term, randomized, controlled studies for assessment of the association between testosterone therapy ( $T$ ) and prostate cancer. The effects of testosterone on prostate cancer growth have been demonstrated in 1940's and withdrawing testosterone by medical castration is one of the mainstream treatments of prostate cancer recently. In this study, the authors investigated whether using testosterone during a 5-year period was associated with an increased risk of high-grade prostate cancer. According to the data from 574 men who had a diagnosis of prostate cancer and history of testosterone use before diagnosis, $\Pi$ had no negative effect on the frequency of high-grade prostate cancer. Although more data are needed, health care providers can begin to consider treating hypogonadal patients who have prostate cancer with $\Pi$ if they are symptomatic.

Emre Bakırcıoğlu, MD 